\title{
Ultrasensitive Chiral Spectroscopy by Dynamical Symmetry Breaking in High Harmonic Generation
}

\author{
Ofer Neufeld, ${ }^{1, * \dagger}$ David Ayuso, ${ }^{2, *}$ Piero Decleva, ${ }^{3}$ Misha Y. Ivanov, ${ }^{2,4,5}$ Olga Smirnova, ${ }^{2,6}$ and Oren Cohen ${ }^{1, *}$ \\ ${ }^{1}$ Physics Department and Solid State Institute, Technion-Israel Institute of Technology, Haifa 32000, Israel \\ ${ }^{2}$ Max-Born Institute for Nonlinear Optics and Short Pulse Spectroscopy, \\ Max-Born-Straße 2A, D-12489, Berlin, Germany \\ ${ }^{3}$ Dipartimento di Scienze Chimiche e Farmaceutiche, Università degli Studi di Trieste, \\ via L. Giorgieri 1, 34127, Trieste, Italy \\ ${ }^{4}$ Department of Physics, Imperial College London, \\ South Kensington Campus, SW72AZ London, United Kingdom \\ ${ }^{5}$ Institute of Physics, Humboldt University Berlin, Newtonstrasse 15, 12489 Berlin, Germany \\ ${ }^{6}$ Technische Universität Berlin, Ernst-Ruska-Gebäude, Hardenbergstraße 36A, D-10623 Berlin, Germany
}

(Received 31 May 2018; revised manuscript received 15 March 2019; published 2 July 2019)

\begin{abstract}
We propose and numerically demonstrate a new chiral spectroscopy method that is based on a universal system-independent mechanism of dynamical symmetry breaking in high harmonic generation (HHG). The proposed technique relies only on intense electric-dipole transitions and not on their interplay with magnetic dipole transitions. The symmetry breaking results in the emission of otherwise "forbidden" harmonics from chiral media (i.e., that are not emitted from achiral or racemic media), yielding a huge, nearly background-free, chiral-achiral signal that is correlated to the magnitude of the medium's enantiomeric excess. The handedness of the medium can be directly detected by measuring the polarization helicity of the emitted harmonics. Moreover, the strength of the "allowed" harmonics (that are not related to symmetry breaking) is chirality independent; hence, they can be used as a reference to probe chiral degrees of freedom within a single measurement. We numerically demonstrate up to $99 \%$ chiral-achiral signal level (normalized difference between the chiral and achiral HHG spectra) from microscopic gas-phase emission using state-of-the-art models for HHG in bromochlorofluoromethane and propylene oxide. We expect the new method to give rise to precise tabletop characterization of chiral media in the gas phase and for highly sensitive time-resolved probing of dynamical chiral processes with femtosecond-to-attosecond temporal resolution.
\end{abstract}

DOI: 10.1103/PhysRevX.9.031002

Subject Areas: Atomic and Molecular Physics, Optics

\section{INTRODUCTION}

Chirality is a fundamental property of asymmetric systems that is abundantly observed in nature. Its analysis and characterization are of tremendous importance in multiple scientific fields, including particle physics, astrophysics, chemistry, and biology. For example, amino acids are generally chiral, as well as DNA and other biologically active molecules [1,2], making molecular chiral

\footnotetext{
*These authors contributed equally to this work.

Corresponding author.

ofern@tx.technion.ac.il

${ }^{\ddagger}$ Corresponding author. oren@technion.ac.il

Published by the American Physical Society under the terms of the Creative Commons Attribution 4.0 International license. Further distribution of this work must maintain attribution to the author(s) and the published article's title, journal citation, and DOI.
}

spectroscopy a necessity for modern drug design $[3,4]$. Chiral spectroscopy is therefore paramount, and novel spectroscopic methods are required to enhance signal strength and resolution, as well as to probe systems with ultrafast chiral dynamics.

Chiroptical techniques that rely on light-matter interactions are often used to detect and characterize molecular chirality [5] and are generally divided into two types: (I) those that require interactions with the magnetic components of the light field, and (II) those that rely solely on the interactions with the electric components of the light field, which are typically much stronger. The latter usually provide higher selectivity, while the former usually provide an easier experimental setup. Accordingly, methods that require magnetic interactions are historically more common and include optical rotation, circular dichroism (CD) absorption spectroscopy, and many more [5,6]. Nevertheless, in the last two decades, electric-dipole-based approaches have been implemented in liquids and surfaces (see Refs. [5,7-9] and references therein) and very recently 
also in the gas phase with several interrelated [10] innovative methods: photoelectron CD (PECD) [5,11-14] and its extension to multiphoton $[15,16]$ and strong-field regimes [17], Coulomb explosion imaging [18,19], and microwave three-wave mixing spectroscopy [20,21]. Another key challenge in chiral spectroscopy is probing ultrafast chiral processes, i.e., time-resolving chiral signals. Femtosecond resolution was obtained with vibrational CD spectroscopy in chiral liquids [22], and PECD [12,23-25] and photoexcitation CD (PXCD) [26,27] in the gas phase. Note that PECD and PXCD are based on angularly resolving the photoelectron spectrum rather than detecting purely optical responses.

Recently, it was also discovered that high harmonic generation (HHG) with a helically polarized pump is chirality sensitive [28-33]. In chiral HHG (cHHG), a chiral medium is irradiated with an intense laser field, and the emission spectrum is measured. cHHG is a very promising technique since it naturally leads to all-optical femtosecond time-resolved signals, and it is accessible in the gas phase by simple tabletop setups [28]. However, current implementations of cHHG exhibit relatively low sensitivity: about 3\% with a somewhat elliptically polarized driver [28] and about $10 \%$ with the so-called bicircular driver $[30,31,33])$. This relatively low sensitivity means, for example, that if the enantiomeric excess of the medium is $20 \%$, then the observed discrimination is about $2 \%$, which may already be within the noise level and therefore not detectable. The main reasons for this relatively low signal are as follows: (1) it is based on interactions involving both the electric and the magnetic components of the light field, and (2) the chiral signal is found by comparing small differences between two or more harmonic spectra that are measured separately, which increases noise. Clearly, a method for $\mathrm{cHHG}$ with much larger chiral signals would open many opportunities, both for characterizing chiral gas media and for exploring ultrafast chirality.

Here, we predict and numerically demonstrate the first cHHG scheme with extreme chiral selectivity, which relies solely on electric-dipole transitions. First, this scheme yields a chiral signal that is embedded in the intensity of otherwise "forbidden" harmonics that are not emitted from achiral media, meaning that the signal is nearly background-free. Second, we show that the medium's handedness is embedded in the emitted harmonic's polarization helicities, which change sign if the medium's handedness is switched. These properties make possible chiral selectivity from a single HHG spectrum (single shot). The new scheme is based on a universal system-independent mechanism of symmetry breaking in chiral media, utilizing the fact that chiral media inherently breaks certain symmetries (e.g., reflections, inversions, dynamical reflections, etc.) that are upheld by the pump field. This idea leads to the emission of a huge chiral signal in the HHG spectrum that is directly correlated to the enantiomeric excess (ee). We analyze this approach and detect the responsible physical mechanism. We present here three feasible realizations of the method based on (A) a static reflection symmetry, (B) a dynamical reflection symmetry, and (C) a dynamical inversion symmetry.

The paper is organized as follows: In Sec. II, we present the concept of our proposal and derive the conditions under which chirality can be detected. In Sec. III, we numerically demonstrate and explore three realizations of cHHG that are based on symmetry breaking of different dynamical symmetries, using a model chiral potential, a quantitative DFT-based model, and a state-of-the-art strong-field model $[30,31,34]$. We conclude and provide an outlook in Sec. IV.

\section{DYNAMICAL SYMMETRY BREAKING IN CHIRAL MEDIA}

We first briefly review dynamical symmetries (DSs) and selection rules in HHG [35] and explain how they can be utilized for chiral spectroscopy. We focus on the microscopic response of a molecule to an intense laser pulse, $\vec{E}(t)$. Since the pulse has a femtosecond duration, the BornOppenheimer approximation is employed, and in this paper, we also employ the dipole approximation (DA). The microscopic Hamiltonian of a single molecule interacting with a laser field is then given in atomic units and in the length gauge by

$$
\begin{aligned}
H_{\Omega}(t)= & -\frac{1}{2} \sum_{j} \vec{\nabla}_{j}^{2}+\frac{1}{2} \sum_{i \neq j} \frac{1}{\left|\vec{r}_{i}-\vec{r}_{j}\right|} \\
& +\sum_{j} V_{\Omega}\left(\vec{r}_{j}\right)+\sum_{j} \vec{E}(t) \cdot \vec{r}_{j},
\end{aligned}
$$

where $H_{\Omega}(t)$ is the time-dependent multi-electron Hamiltonian, $\vec{r}_{j}$ is the coordinate of the $j$ th electron, $\vec{\nabla}_{j}^{2}$ is the Laplacian operator with respect to $\vec{r}_{j}, V_{\Omega}(\vec{r})$ is the molecular potential, and $\Omega$ represents the molecular orientation (as that of a rigid body). The Hamiltonian in Eq. (1) describes the interaction of an oriented molecule with a laser pulse, which, due to the nonlinear laser-matter interaction [the rightmost term in Eq. (1)] generates new harmonic frequencies. Quantum mechanically, the emitted harmonics are calculated as the second-order time derivative of the molecular-induced polarization:

$$
\vec{P}_{\Omega}(t)=-\left\langle\Psi_{\Omega}(\mathrm{t})|\vec{r}| \Psi_{\Omega}(\mathrm{t})\right\rangle,
$$

where $\Psi_{\Omega}(\mathrm{t})$ is the full multi-electron wave function for the orientation $\Omega$, and the integration is performed over all electronic and spin coordinates. The emitted harmonic spectrum is extremely sensitive to the presence of symmetries in $H_{\Omega}$. For example, only odd harmonics are emitted if $H_{\Omega}$ is invariant under a half-wave rotational DS [36], and only circularly polarized harmonics are emitted if $H_{\Omega}$ is invariant under an $n$-fold rotational DS 
(for $n>2$ ) [37-39]. More generally, selection rules are derived as constraints that are a consequence of the invariance of $H_{\Omega}$ with respect to a unitary spatiotemporal transformation (see Ref. [35], which presents a general derivation through group theory for DSs).

When the media is nonoriented, the laser pulse interacts with all possible rigid-body orientations of the molecule uniformly. Therefore, the induced polarization from all orientations should be summed:

$$
\vec{P}_{\text {tot }}(t)=\int \vec{P}_{\Omega}(t) d \Omega
$$

The interaction is described by an effective Hamiltonian for the orientation-averaged ensemble, $H(t)$, which exhibits a higher symmetry than $H_{\Omega}(t)$. As a consequence, many of the molecular properties are "washed out" in HHG experiments. For instance, HHG from randomly oriented molecular $\mathrm{SF}_{6}$ gas and atomic argon gas driven by the same linearly-polarized or bi-circular pump result in identical selection rules [40] because, in both cases, the orientationaveraged molecular potential is spherically symmetric. It is important to note that if the orientation-averaged molecular potential is spherically symmetric (as in achiral media), then selection rules arise only as a consequence of the DSs of the driving laser and not of the microscopic medium. Accordingly, in spherically symmetric media, one may engineer a desired spectral selection rule by wisely choosing the pump-beam geometry.

We now describe the rational of our proposal. Let us consider a chiral ensemble of randomly oriented (chiral) molecules and an achiral ensemble that could be a medium of randomly oriented achiral molecules or a racemic mixture (we do not consider oriented molecular media). Both ensembles are invariant under rotations [i.e., $S O(3)$ ], as they are isotropic. However, only the achiral ensemble is invariant under reflections and inversions [i.e., under $O(3)$ ] since chiral media, by definition, cannot exhibit reflection and inversion symmetry elements (note that inversion is a product of a reflection about a plane and a $\pi$ rotation around the axis normal to that plane). We can use this property in order to distinguish between the ensembles and generate a purely chiral high harmonic signal. To achieve this separation, we suggest using pump fields that (i) generate bright high harmonics, (ii) exhibit a reflection- or inversionrelated DS that leads to forbidden harmonic selection rules in achiral media (easy to observe) that are broken in chiral media, and (iii) do not exhibit other DSs that also lead to the same selection rule but are not broken by chiral media (such as rotational DSs). To elucidate this last requirement, consider the following counterexample: Within the DA, a single monochromatic beam (linearly, elliptically, or circularly polarized) exhibits a dynamical inversion symmetry that forbids even harmonic generation [35]. Thus, one may expect to measure even harmonics from chiral media driven by such a field. However, monochromatic fields also uphold trivial $180^{\circ}$ rotational DSs around their propagation axis that are not broken by chiral media, and lead to the same selection rule. Consequently, the propagating HHG emission from a single monochromatic pump beam cannot detect chirality within the DA.

In this paper, we present three representative HHG geometries that uphold these requirements. Each example utilizes one of the following three DSs:

$$
\begin{gathered}
\hat{\sigma}_{x z}, \\
\hat{Z}=\hat{\sigma}_{x z} \cdot \hat{\tau}_{2}, \\
\hat{F}=\hat{\imath} \cdot \hat{\tau}_{2} .
\end{gathered}
$$

We follow the notation in Ref. [35], where $\hat{\sigma}_{i j}$ represents reflection about the $i j$ plane, $\hat{\tau}_{2}$ represents temporal translation by half of the fundamental period $(T)$, and $\hat{\imath}$ represents spatial inversion. Hence, Eq. (4) describes a "static" space-only reflection transformation about the $x z$ plane $(y \rightarrow-y), \hat{Z}$ in Eq. (5) is a dynamical reflection symmetry about the $x z$ plane $(y \rightarrow-y, t \rightarrow t-T / 2)$, and $\hat{F}$ in Eq. (6) is a dynamical inversion symmetry $(\vec{r} \rightarrow-\vec{r}$, $t \rightarrow t-T / 2$ ). The three DSs in Eqs. (4)-(6) lead to different selection rules on the emitted harmonic spectrum from the spherically symmetric ensemble: $\hat{\sigma}_{x z}$ results in forbidden $y$-polarized $\mathrm{HHG}$ emission, $\hat{Z}$ results in forbidden $x$-polarized odd harmonic emission and forbidden $y$-polarized even harmonic emission [35], and $\hat{F}$ results in forbidden even harmonic emission [35] (see Appendix A. 5 for details). These symmetries are all upheld by randomly oriented achiral media but broken by chiral media, causing an emission of a background-free (up to noise) chiral signal in the form of new harmonics, where the medium's handedness can be retrieved by measuring the harmonic's helicities.

\section{CHIRALITY-SENSITIVE HHG SPECTROSCOPY BASED ON DYNAMICAL SYMMETRY BREAKING}

In this section, we present three schemes for chiral spectroscopy based on cHHG symmetry breaking using different pump-beam geometries, where each one upholds one of the symmetries in Eqs. (4)-(6). We numerically explore these schemes by (i) solving the time-dependent Schrödinger equation (TDSE) for a model chiral potential within the single active electron approximation, (ii) with a DFT-based model for bromochlorofluoromethane (for details, see Appendixes A. 1-A. 4), and (iii) using a strongfield model for propylene oxide (for details, see Appendix A. 7).

\section{A. "Static" reflection symmetry breaking}

We start with the simplest case of the space-only reflection symmetry, $\hat{\sigma}_{x z}$ in Eq. (4). In order to engineer 
(a) (A) scheme illustration

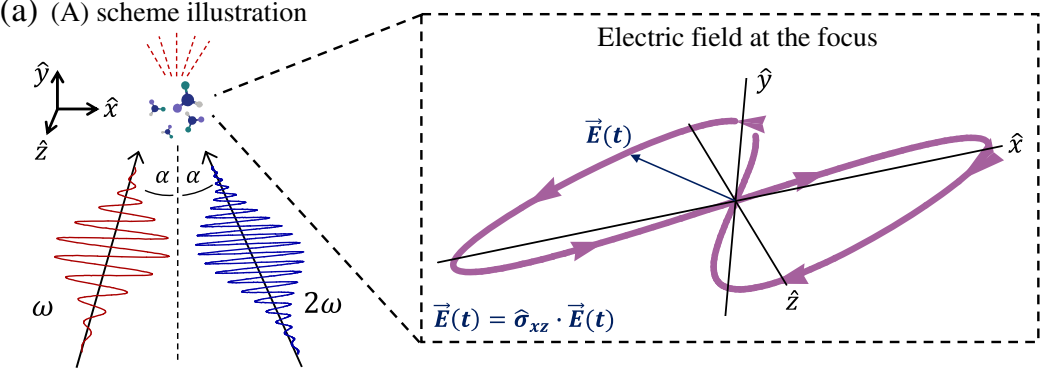

(b) $y$-polarized harmonic spectrum

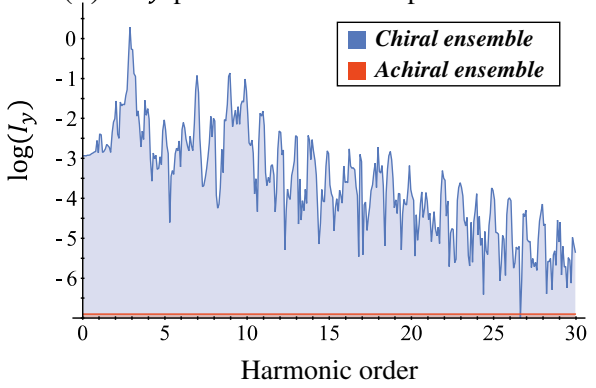

(c)

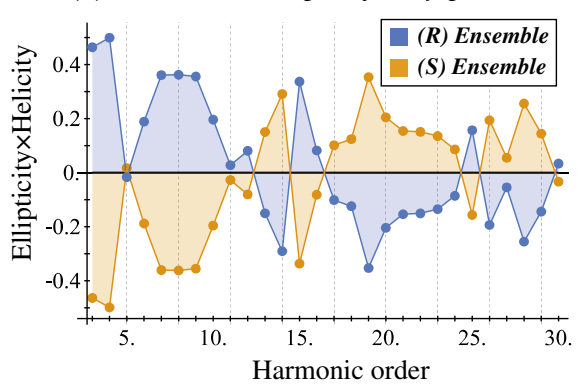

(d) $\quad y$-polarized harmonic yield

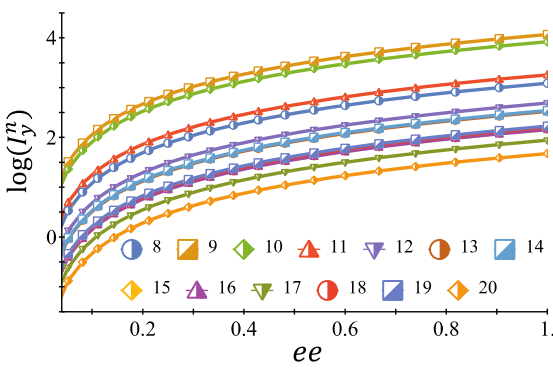

FIG. 1. Static reflection symmetry-breaking-based chirality detection in HHG: Numerical model potential results. (a) Illustration of scheme (A) with the pump field from Eq. (7). The inset shows the 3D Lissajou plot of the microscopic electric field vector in the focus (though, in this case, the field is $2 \mathrm{D}$ and is contained in the $x z$ plane), which exhibits a static reflection symmetry (arrows along the Lissajou represent the direction of time). (b) Numerically calculated $y$-polarized HHG emission from the chiral and achiral ensembles ( $\psi_{6}$ in model potential) for $\lambda=900 \mathrm{~nm}, \mathrm{I}_{\max }=6.5 \times 10^{13} \mathrm{~W} / \mathrm{cm}^{2}, \phi=0, \Delta=1, \alpha=15^{0}$, and a trapezoidal envelope with fouroptical-cycle long turn-on/off and four-optical-cycle long flattop. In the chiral ensemble, all harmonic orders have $y$-polarized components (both even and odd), while there is no $y$-polarized harmonic emission from the achiral ensemble. (c) Calculated harmonic ellipticities in the $x y$ plane from $(\mathrm{R})$ and $(\mathrm{S})$ ensembles - the helicity changes sign with the medium's handedness. (d) Integrated $y$-polarized yield per harmonics $8-20$ vs $e e$ in log scale.

a laser field that exhibits this symmetry and upholds the desired requirements, one must consider noncollinear HHG schemes [41-43]. Any collinear scheme necessarily exhibits other DSs that prevent chiral sensitivity in the propagating modes. We therefore consider the following $\omega-2 \omega$ noncollinear HHG scheme:

$\vec{E}_{A}(t ; \vec{r})=A(t) E_{0} \operatorname{Re}\left\{e^{i\left(\omega t-\vec{k}_{1} \cdot \vec{r}+\phi\right)} \hat{e_{1}}+\Delta e^{i\left(2 \omega t-2 \vec{k}_{2} \cdot \vec{r}\right)} \hat{e_{2}}\right\}$,

where $\omega$ is the optical frequency, $\vec{k}_{1}=k(\sin (\alpha) \hat{x}-\cos (\alpha) \hat{\mathrm{z}})$ is the wave vector for the $\omega$ beam, $\vec{k}_{2}=-k(\sin (\alpha) \hat{x}+$ $\cos (\alpha) \hat{z})$ is the wave vector for the $2 \omega$ beam, $\hat{e}_{1}=$ $\cos (\alpha) \hat{x}+\sin (\alpha) \hat{z}, \quad \hat{e}_{2}=\cos (\alpha) \hat{x}-\sin (\alpha) \hat{z}, \alpha$ is half the opening angle between the two beams in the $x z$ plane, $k$ is the magnitude of the wave vector related to the optical frequency, $A(t)$ is a dimensionless envelope function, $E_{0}$ is the field amplitude, $\Delta$ is the amplitude ratio between the beams, and $\phi$ is an arbitrary phase. The spatial envelope of the field in Eq. (7) is neglected in the DA (plane-wave treatment). Overall, $\vec{E}_{A}(t, \vec{r})$ comprises a linearly polarized fundamental $\omega$ beam propagating in the $x z$ plane, and a linearly polarized $2 \omega$ beam propagating in tandem. Both beams are polarized in the $x z$ plane, and their coherent superposition generates a microscopic field that is invariant under $\hat{\sigma}_{x z}$, independently of the values of the parameters $\phi$ and $\Delta$ [see Fig. 1(a)]. This field is not invariant under any other DS (except $\hat{\sigma}_{x z}$ ); hence, all harmonic orders are allowed with general elliptical polarization in the $x z$ plane, while emission of $y$-polarized harmonics is forbidden yet should occur in chiral media because of reflection symmetry breaking. The microscopic pump field may differ in areas far from the beam center, but this does not hamper the scheme since $\hat{\sigma}_{x z}$ symmetry holds for any phase or intensity ratio between the $\omega$ and $2 \omega$ beams.

Figure 1(b) shows the microscopic $y$-polarized $\mathrm{HHG}$ emission from the model potential chiral ensemble, where $y$-polarized harmonics survive orientation averaging. In contrast, the $y$-polarized spectrum from the racemic mixture is zero; i.e., $y$-polarization components do not survive orientation averaging. This occurs within the DA because the partner enantiomers in the ensemble acquire an opposite phase factor - an (R) enantiomer driven by an orientation $\Omega$ is equivalent to an (S) enantiomer driven by the reflected orientation $\Omega^{*}$, up to a minus sign for the $y$-polarized emission, because the pump is reflection invariant. Hence, in the racemic mixture (achiral ensemble), these contributions exactly interfere destructively (this is schematically illustrated in Fig. 2). In other words, there exists a mapping between the HHG emission from the (R) and (S) ensembles due to the mirror symmetry in the pump. On the other hand, 


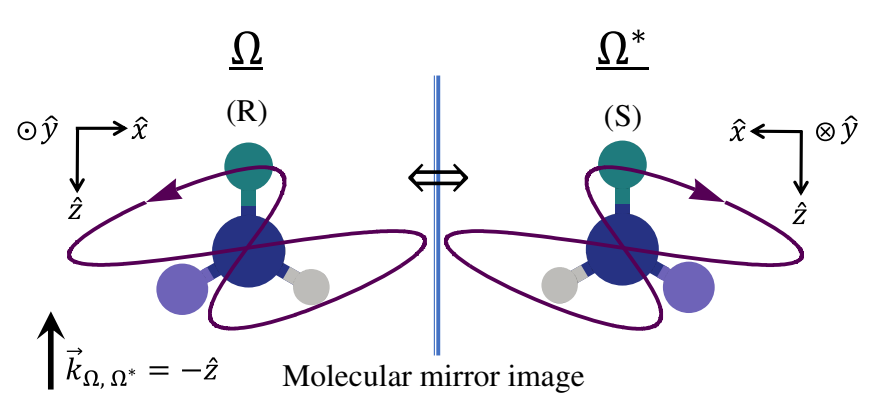

FIG. 2. Mechanism for destructive interference in HHG from racemic mixtures illustrated in scheme (A): For every orientation $\Omega$ of enantiomer (R), there exists a DA-equivalent orientation $\Omega^{*}$ for enantiomer (S) due to the reflection symmetry of the pump and the mirror image relation between the $(\mathrm{R})$ and $(\mathrm{S})$ enantiomers. These two orientations exactly destructively interfere for $y$-polarized HHG emission in the achiral ensemble (which differs by a sign between the two enantiomers for every orientation pair $\Omega, \Omega^{*}$ ). However, for nonracemic mixtures (nonzereo $e e$ ), the destructive interference is incomplete, leading to $y$-polarized HHG emission. The purple Lissajou plot shows the overall vector pump field in the dipole approximation, and $\Omega$ and $\Omega^{*}$ denote the equivalent molecular orientations of $(\mathrm{R})$ and $(\mathrm{S})$ enantiomers that lead to destructive interference.

there is no such mapping in the chiral ensemble because, by definition, there is no orientation for which the molecule can be superposed onto its mirror image. In-between these two extreme cases (for $0<|e e|<1$ ), there is partial destructive interference for the $y$-polarized emission, causing the yield to depend on the value of the ee. Importantly, the $y$-polarized chiral emission is perpendicular to the plane of incidence and can therefore conserve momentum and propagate.

This interference-based mechanism means that the cHHG spectra from partner chiral ensembles have identical spectral intensities and are distinguishable in the DA only through the global $\pi$ phase shift- the chiral signal emitted with $y$-polarization changes sign with the medium's handedness, while the $x$-polarized emission does not $[20,26]$. This global phase shift is directly reflected in the emitted harmonics ellipticities, which change their handedness between (R) and (S) media as shown in Fig. 1(c). The ellipticities of some harmonics reach as high as \pm 0.5 ; hence, they can be used to characterize the medium's handedness in a single-shot measurement (a single-perturbative or nonperturbative harmonic order is enough). Furthermore, we note that this electric-dipolebased response does not require interference between different HHG channels because it is a result of the coherent interference of emissions from different molecular orientations, making our scheme general and system independent (the only prerequisite is that harmonics are generated). It is also worth mentioning that the pump field in Eq. (7) leads to chiral selectivity even though it is comprised of linearly polarized beams (that are individually achiral). This is because the coherent superposition of the beams generates an overall helical field with nonzero optical chirality [44-46], analogously to the scheme presented in Ref. [20].

Figure 1(d) presents the integrated intensity of the $y$-polarized emission per harmonic order vs the medium's $e e$, which is numerically calculated by coherently adding the polarization from both enantiomers and renormalizing:

$$
\vec{P}_{\mathrm{tot}}^{e e}(t)=\frac{(1+e e) \vec{P}_{\mathrm{tot}}^{(\mathrm{R})}(t)+(1-e e) \vec{P}_{\mathrm{tot}}^{(\mathrm{S})}(t)}{2},
$$

where $e e$ is the enantiomeric excess. The intensity of the $y$ emission increases parabolically as a function of the $e e$ for $e e \in[0,1]$, and it results in extremely large chiral/achiral signals that are effectively single shot because the intensity of the "forbidden" emission can always be normalized with respect to the "allowed" emission that is chirality insensitive. The spectroscopy technique can therefore be used for single-shot high-resolution ee measurements. We define the chiral/achiral signal between the two ensembles per harmonic order $n$ as

$$
S_{n}=\frac{I_{n}^{e e=1}-I_{n}^{e e=0}}{I_{n}^{e e=1}+I_{n}^{e e=0}},
$$

which ranges from 0 to $100 \%$ (note that $S_{n}$ discriminates between the chiral and achiral ensembles, not the (R) and (S) ensembles, and differs by a factor of 2 from standard definitions for chiral signals). A similar measure can be defined through the HHG helicity in the $x y$ plane to distinguish the $(\mathrm{R}) /(\mathrm{S})$ ensembles:

$$
H_{n}=h_{n}^{e e=1}-h_{n}^{e e=-1},
$$

where $h_{n}$ is the helicity of the $n$th harmonic. The product of $H_{n}$ and $S_{n}$ provides the standard (R)/(S) chiral selectivity that ranges from $-200 \%$ to $200 \%$ per harmonic order. Other effective chiral observables can also be defined. For example, one may average $S_{n}$ over all harmonic orders to increase the signal-to-noise ratio. Numerically, we find $100 \%$ disparity between chiral and achiral media for the $y$-polarized emission, and $(\mathrm{R}) /(\mathrm{S})$ handedness selectivity through the helicity of the emitted harmonics. The maximal signal is attributed to the fact that $\hat{\sigma}_{x z}$ is an exact symmetry of our model (this symmetry has no temporal part and is therefore not broken by the finite duration of the laser pulse, or by ionization). In reality, magnetic interactions that are neglected in this analysis may increase the noise in accordance with standard $\mathrm{cHHG}(\sim 5 \%)$ [30], possibly slightly reducing the signal.

\section{B. Dynamical reflection symmetry breaking}

We now consider symmetry breaking of the dynamical reflection symmetry in Eq. (5). In order to engineer a laser field that exhibits $\hat{Z}$ symmetry and upholds the desired requirements, we consider the following bichromatic noncollinear $\omega-2 \omega$ geometry: 
(a) (B) scheme illustration
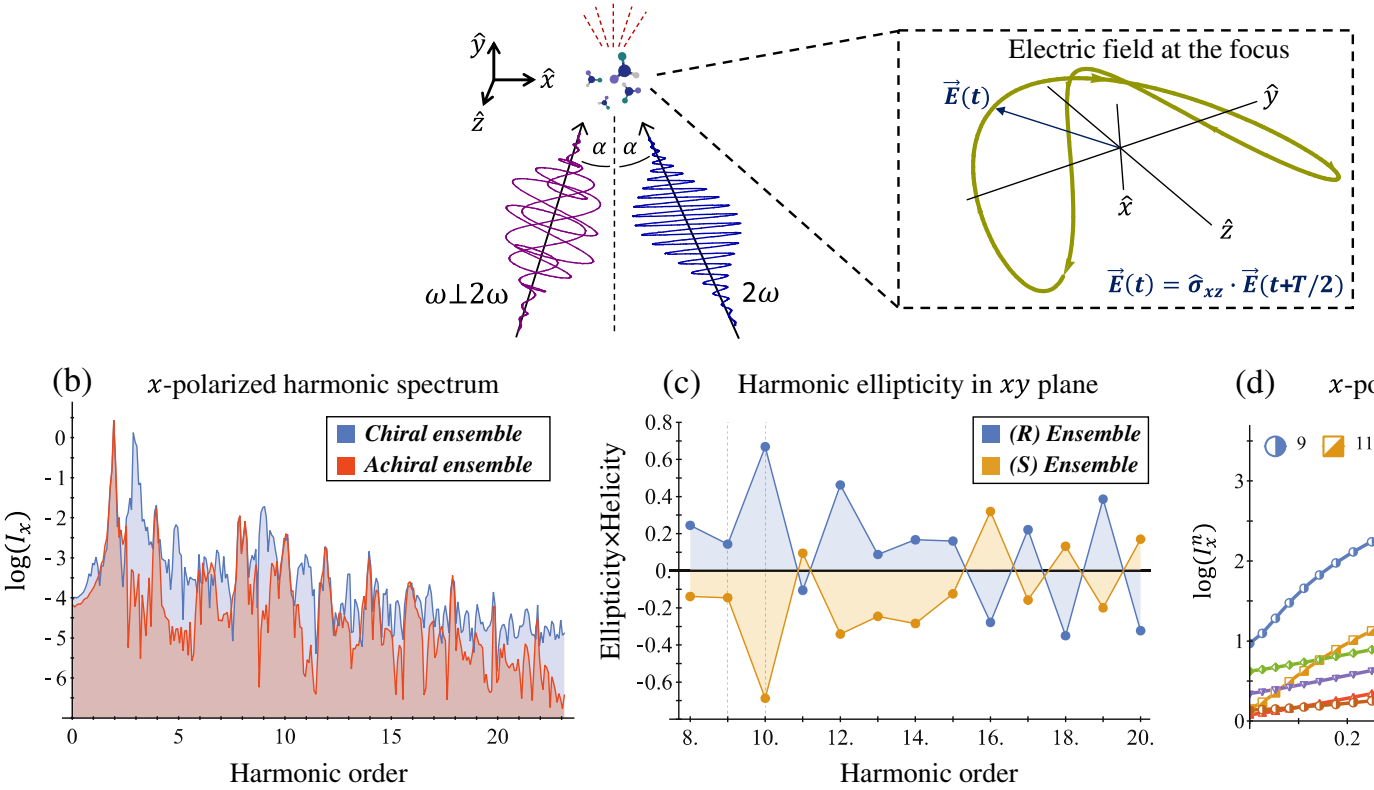

(c) Harmonic ellipticity in $x y$ plane

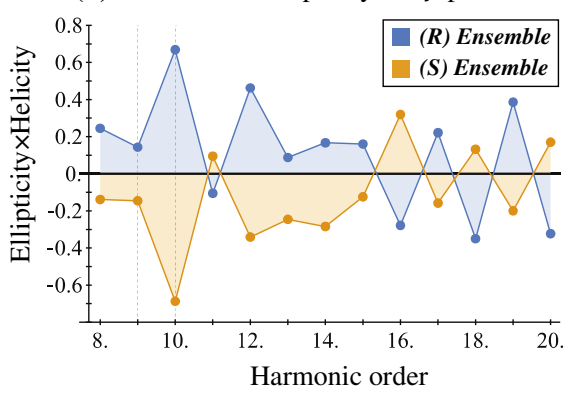

(d) $\quad x$-polarized odd harmonic yield

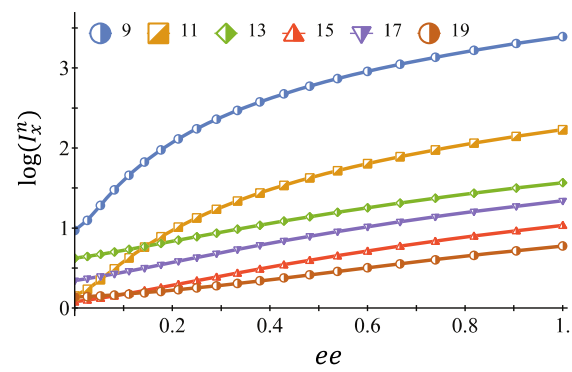

FIG. 3. Dynamical reflection symmetry-breaking-based chirality detection in HHG: Numerical model potential results. (a) Illustration of scheme (B) with the pump field in Eq. (11). The inset shows the 3D Lissajou plot of the microscopic electric field vector in the focus, which exhibits a dynamical reflection symmetry (arrows along the Lissajou represent the direction of time). (b) $x$-polarized high harmonics emitted from the chiral and achiral ensembles ( $\psi_{6}$ in the model potential), for fundamental wavelength $\lambda=900 \mathrm{~nm}$, $I_{\max }=4.2 \times 10^{13} \mathrm{~W} / \mathrm{cm}^{2}, \phi=\pi / 9, \Delta_{1}=1.1, \Delta_{2}=0.97, \alpha=15^{0}$, and a trapezoidal envelope with four-cycle turn-on/off and fourcycle flattop. (c) Calculated harmonic ellipticities in the $x y$ plane from (R) and $(\mathrm{S})$ ensembles - the helicity changes sign with the medium's handedness. (d) Integrated power of the $y$-polarized odd harmonics 9-19 vs the ee in log scale.

$$
\begin{aligned}
\vec{E}_{B}(t ; \vec{r})= & A(t) E_{0} \operatorname{Re}\left\{e^{i\left(\omega t-\vec{k}_{1} \cdot \vec{r}+\phi\right)} \hat{y}+\Delta_{1} e^{i\left(2 \omega t-2 \vec{k}_{1} \cdot \vec{r}\right)} \hat{e_{1}}\right. \\
& \left.-i \Delta_{2} e^{i\left(2 \omega t-2 \vec{k}_{2} \cdot \vec{r}\right)} \hat{e_{2}}\right\}
\end{aligned}
$$

where $\Delta_{1,2}$ are relative amplitude ratios between the beams, and other symbols are as previously defined. Note that $\vec{E}_{B}(t ; \vec{r})$ is comprised of a $2 \omega$ beam linearly polarized in the $x z$ plane and propagating with a wave vector $\vec{k}_{2}$, and a bichromatic cross-linearly polarized $\omega-2 \omega$ beam propagating with a wave vector $\vec{k}_{1}$ [see Fig. 3(a) for illustration]. The superposition of these noncollinear bichromatic beams is invariant in the DA under $\hat{Z} \mathrm{DS}$, independently of the phase relation $\phi$ and amplitude ratios $\Delta_{1,2}$, even though the microscopic field is a 3D vector field [see inset in Fig. 3(a)]. This results from the fact that the $\omega$ wave is polarized along the $y$ axis and is always transverse to the $2 \omega$ waves that are elliptically polarized in the $x z$ plane.

Figure 3(b) presents the intensity of the emitted harmonics polarized along the $x$ axis from the model potential chiral and achiral ensembles. As seen, $x$-polarized odd harmonics do not survive orientation averaging in the achiral ensemble due to the selection rules imposed by $\hat{Z}$ DS. However, they do survive orientation averaging in the chiral ensemble. The mechanism for this effect is identical to that in the static symmetry-breaking case described in Fig. 2, though here the signal-to-noise ratio is lower since $\hat{Z}$ DS is also somewhat broken by the finite duration of the pulse and by ionization in the medium (i.e., some $x$-polarized odd harmonics can also be emitted from the achiral ensemble). Similarly to scheme (A), the mapping between HHG emission from (R) and (S) ensembles leads to a global $\pi$ phase shift for the chiral signal, while the achiral emission is identical between the $(\mathrm{R})$ and $(\mathrm{S})$ ensembles. Consequently, the ellipticity of the odd harmonics [see Fig. 3(c)] in the $x y$ plane changes sign with the medium's handedness (because $y$-polarized odd harmonics constitute an achiral signal). Note that in Fig. 3(b), the intensity of the even allowed harmonics does not depend on the chirality and can be used as a normalization to make the scheme single shot. Figure 3(d) presents the integrated power of $x$-polarized odd harmonics vs the $e e$, for $e e \in$ $[0,1]$, showing that this scheme is highly sensitive over a wide spectral range, with the same parabolic scaling as in scheme (A). We find a disparity of $99 \%$ between the chiral and achiral ensembles through the integrated $x$-polarized odd harmonic power, though magnetic interactions will slightly decrease this signal (by about 5\% according to standard cHHG [30]).

It is worthwhile mentioning that $\hat{Z}$ DS also leads to forbidden $y$-polarized even harmonic emission selection rules [35]. As a result, $y$-polarized even harmonics also constitute a nearly background-free chiral signal. Their ellipticity also changes sign with the medium's handedness, 


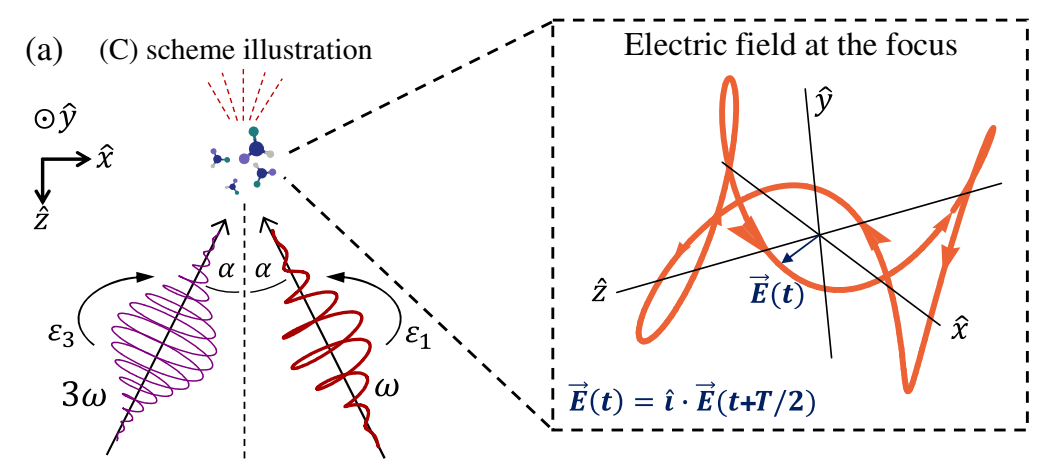

(b) Emitted harmonic spectrum

(c) Chiral/achiral signal vs. $\alpha$
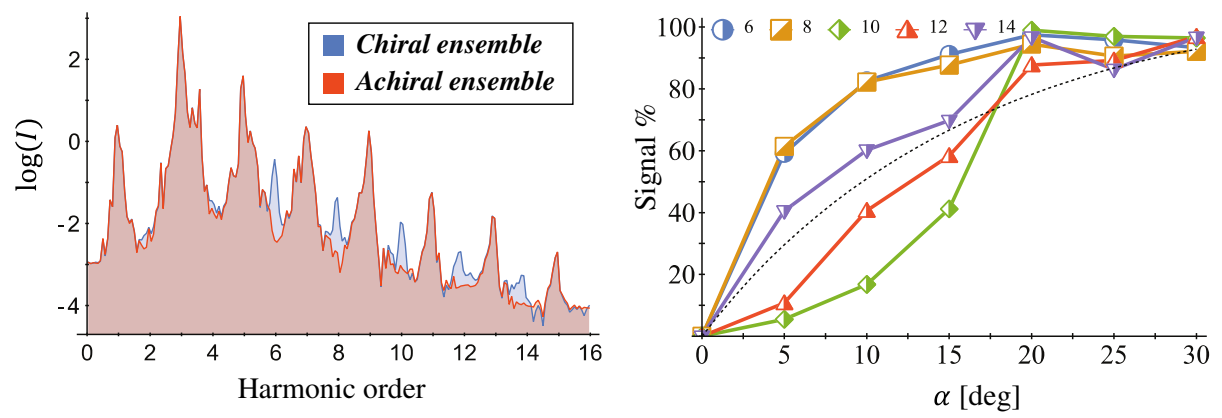

FIG. 4. Dynamical inversion symmetry-breaking-based chirality detection in HHG: Numerical model potential results. (a) Illustration of scheme (C) with the pump field given in Eq. (12). The inset shows the 3D Lissajou plot of the microscopic electric field vector in the focus, which exhibits a dynamical inversion symmetry (arrows along the Lissajou represent the direction of time). (b) High harmonics emitted from the chiral and achiral ensembles ( $\psi_{15}$ in the model potential), fundamental wavelength $\lambda=700 \mathrm{~nm}, I_{\max }=10^{13} \mathrm{~W} / \mathrm{cm}^{2}$, $\phi=\pi / 2, \Delta=1, \varepsilon_{1}=0.4, \varepsilon_{3}=0.3, \alpha=20^{\circ}$, and a trapezoidal envelope with four-cycle turn-on/off and five-cycle flattop. (c) Chiral/ achiral signal from $y$-polarized even harmonics as a function of $\alpha$ for the same conditions as in panel (b). The dashed line represents an approximately $\sim \sin (2 \alpha)$ trend line for the harmonic yield, roughly representing the increase of the chiral signal with $\alpha$.

since the $x$-polarized even harmonics are achiral, and can be used as a single-shot reference [see Fig. 3(c), which presents the ellipticities of both even and odd harmonics]. This scheme, therefore, favorably leads to chiral signal emission in all polarization axes, unlike scheme (A).

\section{Dynamical inversion symmetry breaking}

We consider a third method for chiral spectroscopy through dynamical inversion symmetry breaking [Eq. (6)] that is driven by the following noncollinear bichromatic $\omega-3 \omega$ beam geometry:

$$
\begin{aligned}
\vec{E}_{C}(t ; \vec{r})= & A(t) E_{0} \operatorname{Re}\left\{e^{i\left(\omega t-\vec{k}_{2} \cdot \vec{r}+\phi\right)}\left(\hat{e_{2}}-i \varepsilon_{1} \hat{y}\right)\right. \\
& \left.+\Delta e^{i\left(3 \omega t-3 \vec{k}_{1} \cdot \vec{r}\right)}\left(\hat{e_{1}}+i \varepsilon_{3} \hat{y}\right)\right\},
\end{aligned}
$$

where $\varepsilon_{1}$ is the ellipticity of the $\omega$ beam, $\varepsilon_{3}$ is the ellipticity of the $3 \omega$ beam, and other symbols are as previously defined [see Fig. 4(a) for illustration]. $\vec{E}_{C}(t ; \vec{r})$ is comprised of two elliptical beams propagating in tandem in the $x z$ plane with carrier frequency ratios $1: 3$, which upholds $\hat{F}$ symmetry for all coordinates along the beam paths, for any ellipticities $\varepsilon_{1,2}$, amplitude ratio $\Delta$, opening angle $\alpha$, and phase $\phi$, as well as in the presence of $\omega$ components in the $3 \omega$ beam.

Figure 4(b) shows that in the model potential, $\hat{F}$ symmetry is broken by the chiral ensemble and leads to the generation of even harmonics, while this symmetry is upheld in the achiral ensemble where even harmonics are forbidden. A disparity of $96 \%$ is obtained in the even harmonic intensities, while odd harmonics provide an achiral reference signal. Similar to schemes (A) and (B), the chiral even harmonic emission is globally phase shifted by $\pi$ when the medium's handedness is switched. However, in this case, there is no achiral emission of even harmonic frequencies; therefore, this phase is difficult to measure, as it is not directly reflected in the harmonic ellipticities. Still, scheme (C) has some advantages because here the chiral signal is nearly background-free regardless of its polarization components. Notably, there are 7 independent degrees of freedom (d.o.f.) in the bichromatic beam $\vec{E}_{C}(t, \vec{r})$, including ellipticities, opening angles, etc., which provide much freedom for optimizing the signal-to-noise ratio. Because of limited computational time, we cannot fully optimize these parameters (each calculation for a single orientation takes about 24 hrs on a Tesla K80 GPU, and many calculations need to be performed to orientation 


\section{Bromochlorofluoromethane (DFT)}
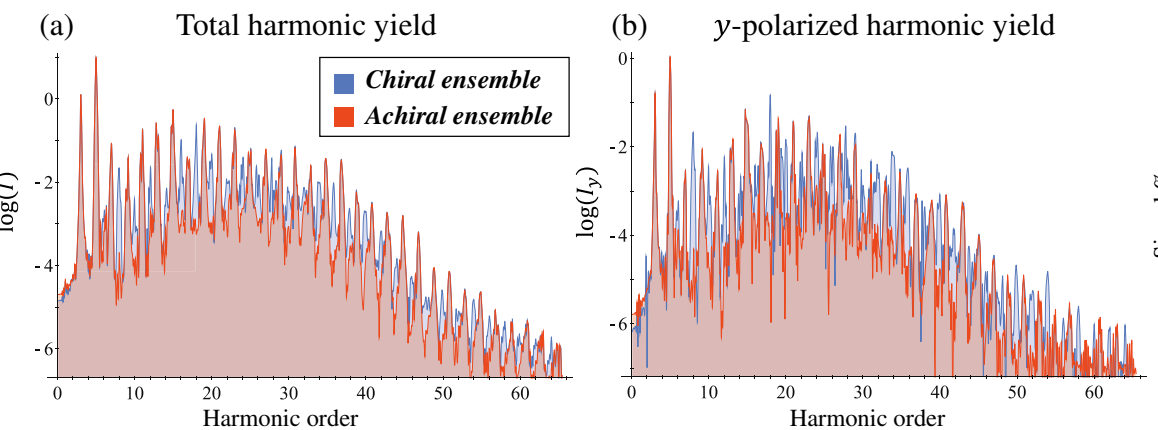

(c) Chiral/achiral signal per harmonic

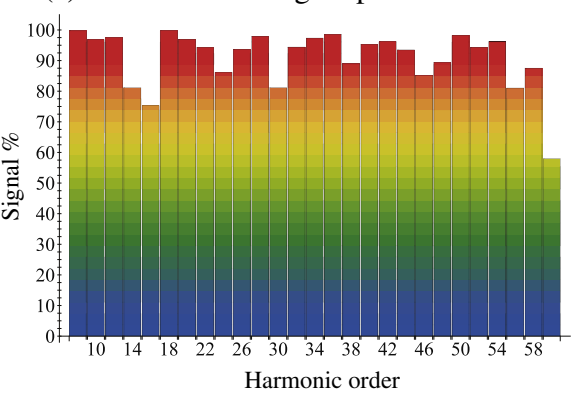

(d)

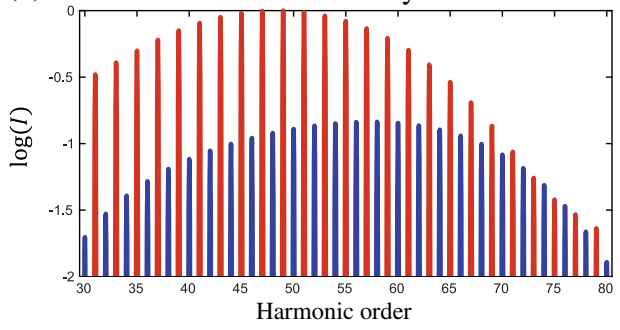

(e)

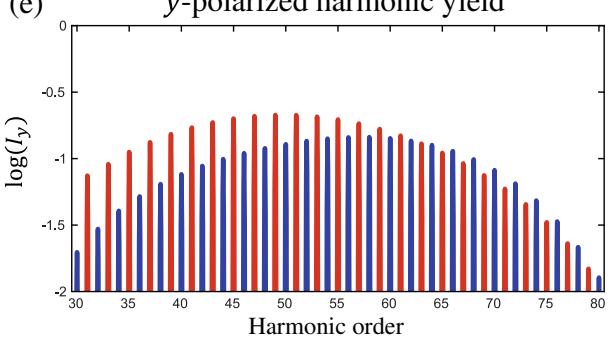

Propylene oxide (strong field)

FIG. 5. Dynamical inversion symmetry-breaking-based chirality detection in HHG from DFT-based calculations for CBrClFH $[(a)-(c)]$ and a molecular strong-field model for $\mathrm{C}_{3} \mathrm{H}_{6} \mathrm{O}[(\mathrm{d}, \mathrm{e})]$. (a) Total orientation-averaged high harmonic signal from the chiral and achiral ensembles of $\mathrm{CBrClFH}$ from a DFT-based model for fundamental wavelength $\lambda=3300 \mathrm{~nm}$, frequency ratios $3 \omega-5 \omega$ in the pump field, $\mathrm{I}_{\max }=1.2 \times 10^{13} \mathrm{~W} / \mathrm{cm}^{2}, \phi=\pi / 2, \Delta=1, \varepsilon_{1}=0.4, \varepsilon_{3}=0.3, \alpha=20^{0}$, and a trapezoidal envelope with four-cycle turnon/off and three-cycle flattop. (b) Same as panel (a) but for a $y$-polarized emitted harmonic signal. (c) Chiral/achiral signal per harmonic order defined through the harmonic integrated $y$-polarized signal, reaching as high as $99 \%$. (d,e) Orientation-averaged harmonic spectra under similar settings to (a)-(c) from a strong-field model for $\mathrm{C}_{3} \mathrm{H}_{6} \mathrm{O}$ but with $\omega-3 \omega$ in the pump field for fundamental wavelength $\lambda=1900 \mathrm{~nm}$, and $\mathrm{I}_{\max }=5 \times 10^{13} \mathrm{~W} / \mathrm{cm}^{2}$.

average both ensembles). Thus, we focus on the most important d.o.f.

Figure 4(d) presents TDSE calculations of the chiral/ achiral signal of several harmonic orders as a function of the opening angle, $\alpha$. For $\alpha \rightarrow 0$, the signal diminishes, which is expected because for $\alpha=0$ the microscopic field is invariant under a $180^{\circ}$ rotational DS that forbids even harmonic generation, and it is not broken by chiral media; hence, the signal diminishes as this DS is approached. The noncollinear scheme is thus necessary to break this rotational DS. The chiral/achiral signal ratio shows an overall increasing trend with the longitudinal pump-field amplitude, which depends linearly on $\sin (\alpha)$. This behavior is complex and different for different harmonic orders, as shown in Fig. 4(d). The trend also applies to schemes (A) and (B) - a noncollinear beam geometry is essential for chiral signal propagation. Similarly, we expect the chiral signal to decrease when any other parameter choices lead to a $180^{\circ}$ rotational DS in Eq. (12), such as $\Delta \rightarrow 0$. Another prominent principle for choosing the values of the free parameters is maximizing the harmonic yield (see Appendix A. 6 for further discussion).

For scheme (C), we also present density-functionaltheory (DFT) based model calculations for the chiral molecule bromochlorofluoromethane $(\mathrm{CBrClFH})$ and molecular strong-field model calculations for the chiral molecule propylene oxide $\left(\mathrm{C}_{3} \mathrm{H}_{6} \mathrm{O}\right)$. Generally, the chiral/ achiral signal may differ in different systems due to dependence on various parameters, including the molecular structure, ionization potential, and other chemical and physical properties. It is therefore useful to perform quantitative calculations on real systems other than the examined model potential. For this purpose, we employ a DFT-based model for $\mathrm{CBrClFH}$ using the real-space gridbased octopus code $[47,48]$ (see Appendix A. 2). Scheme (C) is realized using $3 \omega-5 \omega$ frequency ratios in the pump field, where $\omega$ corresponds to $3300-n m$ light $[\hat{F}$ symmetry may also be obtained by any pair of beams with frequency ratios $\omega_{1}: \omega_{2}$ that, in reduced fractional form, are odd integers, where for a $1: 1$ ratio from monochromatic beams, scheme $(\mathrm{C})$ reduces to $(\mathrm{A})]$. Results are presented in Figs. 5(c), and confirm the model potential calculationsthe disparity between the chiral and achiral ensembles reaches as high as 99\%, and the odd harmonic signal remains achiral, corroborating the generality of our approach. Moreover, a molecular strong-field model is employed in $\mathrm{C}_{3} \mathrm{H}_{6} \mathrm{O}$ (see Appendix A. 7) as is presented in Figs. 5(d) and 5(e); it supports previous analytical and 
numerical results (though here the signal reaches exactly $100 \%$ because the strong-field model is perfectly time periodic).

It is worth noting that the chiral/achiral signal obtained from $\mathrm{CBrClFH}$ is larger than the one obtained from the model potential under a similar setting (the mean intensity ratio of chiral even harmonics to their reference achiral neighboring odd harmonics is larger by a factor of 3 in $\mathrm{CBrClFH}$ than in the model potential). This naturally reflects the degree of chirality of these two systems, where the model potential exhibits a relatively low chirality compared to that of a real molecule (see Appendix A. 1).

\section{CONCLUSIONS AND OUTLOOK}

To summarize, we proposed and numerically demonstrated a novel system-independent approach for cHHG that is based on dynamical symmetry-breaking spectroscopy (and not on the detailed mechanism of the HHG process). Our approach relies solely on intense electricdipole transitions and not on the interplay of both magnetic and electric dipoles as in all other cHHG schemes [2831,33 ], making it strong and highly robust. We explored different feasible realizations for the scheme by systematically engineering pump fields with various dynamical symmetry properties that are broken in chiral media. This approach is general and can be applied via an infinite set of geometries [i.e., our suggested schemes (A), (B), and (C) are representative.] Remarkably, the technique results in extremely high chiral/achiral sensitivity of up to $99 \%$, which outperforms, by far, the ability of previous cHHG techniques to discern chiral media [28-33]. It also allows direct access to the medium's handedness by measuring the harmonic's helicities, which change sign when the medium's handedness is switched. Notably, here we predicted and analyzed cHHG based on electric-dipole interactions microscopically. In a future paper [49], we will show that cHHG based on electric-dipole interactions can provide very large chiral sensitivity, both in the microscopic response and in the macroscopic emission. Furthermore, in Ref. [49] we will introduce a new type of chiral light and show that it allows discriminating between opposite enantiomers at the level of total signal intensities.

At first glance, the conditions at which chiral sensitivity can be observed within the electric-dipole approximation may seem arbitrary. For instance, schemes (B) and (C) only differ by beam-frequency ratios, but one allows (R)/(S) sensitivity while the other only allows chiral/achiral sensitivity. Nonetheless, these seemingly minute differences are a manifestation of the underlying dynamical symmetry group of the pump beam [35] and of which symmetry elements are included or excluded from it. We believe that this comprehensive analysis will be useful in many areas of physics and chemistry. Beyond the general formalism, our work paves the way for tabletop, highly sensitive, chiral spectroscopy in the gas phase, and for single-shot, femtosecond-resolved, ultrafast spectroscopy of chiral processes (including weak chirality). We also believe that this work will advance HHG spectroscopy for other physical phenomena, including magnetic interactions, spin-orbit effects, and more, by implementing an analog approach.

\section{ACKNOWLEDGMENTS}

This work was supported by the Israel Science Foundation (Grant No. 1781/18) and the Israeli Center of Research Excellence "Circle of Light" supported by the I-CORE Program of the Planning and Budgeting Committee and the Israel Science Foundation, and the Wolfson Foundation. O.N. gratefully acknowledges the support of the Israel Academy of Sciences and Humanities. D. A. and O.S. acknowledge support from the DFG SPP 1840 "Quantum Dynamics in Tailored Intense Fields" and DFG Grant No. SM 292/5-2. O.S. acknowledges support from the MEDEA Project. The MEDEA Project has received funding from the European Union's Horizon 2020 Research and Innovation Programme under the Marie Sklodowska-Curie Grant Agreement No. 641789.

\section{APPENDIX: A NUMERICAL DETAILS AND ADDITIONAL DATA}

This appendix contains details on the methodology used in numerical calculations presented in the main text, as well as additional results. The file is ordered as follows: Appendix A. 1 describes the model chiral potential. Appendix A. 2 describes the DFT-based model used for bromochlorofluoromethane ( $\mathrm{CBrClFH})$. Appendix A. 3 describes the integration details of the TDSE performed for both models. Appendix A. 4 describes the details of the orientation averaging and its convergence. Appendix A. 5 derives the selection rules for the dynamical symmetries (DSs) used in schemes (A)-(C), following Ref. [35]. Appendix A. 6 provides additional numerical DFT-based model calculations for $\mathrm{CBrClFH}$ in different parameter settings for scheme (C). Appendix A. 7 describes the molecular strong-field model used for propylene oxide.

\section{Model chiral potential-Numerical details}

A chiral model potential is described by the following expression:

$$
\begin{aligned}
V(\vec{r})= & -\frac{z_{1}}{\sqrt{\left(\vec{r}-\vec{r}_{1}\right)^{2}+a}}-\frac{z_{2}}{\sqrt{\left(\vec{r}-\vec{r}_{2}\right)^{2}+a}} \\
& -\frac{z_{3}}{\sqrt{\left(\vec{r}-\vec{r}_{3}\right)^{2}+a}}-\frac{z_{4}}{\sqrt{\left(\vec{r}-\vec{r}_{4}\right)^{2}+a}},
\end{aligned}
$$

where $z_{1,2,3,4}=1.5,1.25,1,0.75$, respectively, $\vec{r}_{1}=0$, $\vec{r}_{2}=-\hat{x}, \vec{r}_{3}=\hat{y}, \vec{r}_{4}=\hat{z}$ bohr, and $a=0.05$ a.u. The isosurface plot for this potential is presented in Fig. 6(a). The enantiomeric atom is found by reflecting along the $y z$ plane (i.e., $\vec{r}_{2}^{*}=\hat{x}$ ). The eigenstates for this potential 

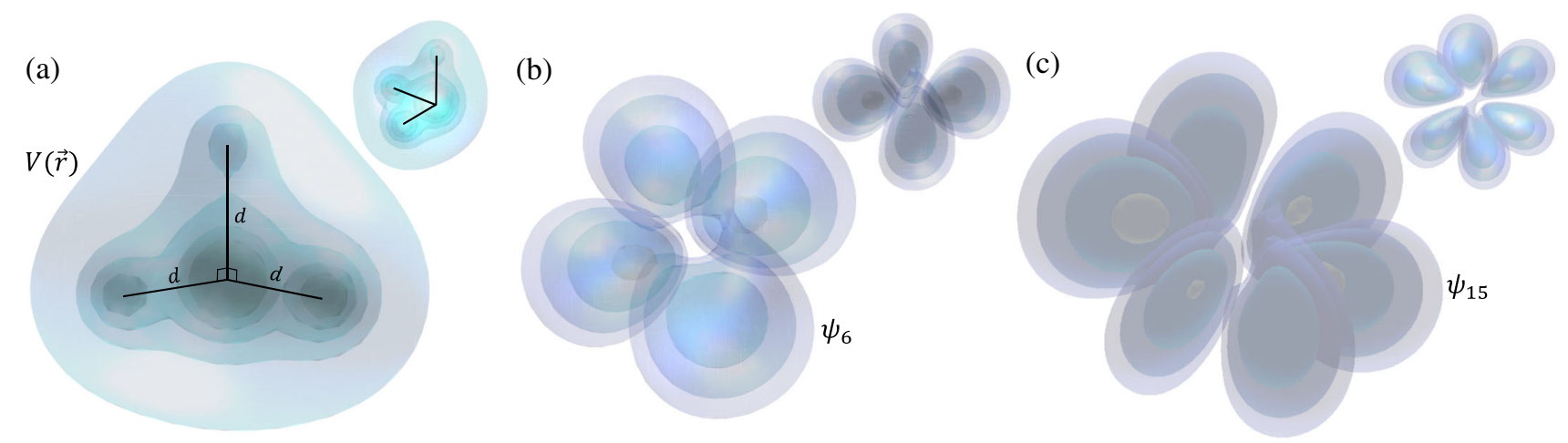

FIG. 6. Isosurface plots for (a) the chiral model potential, (b) the sixth orbital, and (c) the $15^{\text {th }}$ orbital. In each subfigure, a second viewpoint is shown in the inset. Isosurfaces for the potential are $s=0.15,0.3,0.4,0.5,0.6 \mathrm{~V}_{\max }$, and for the orbitals, they are $s=0.05$, $0.1,0.25,0.75|\psi|_{\max }{ }^{2}$. In diagram (a), the lines represent the molecular backbone for the model potential, where $d=1$ a.u.

are found by complex time propagation of the TDSE (as detailed in A. 3) and a Gram-Schmidt algorithm, implemented to a self-consistency convergence $<10^{-8}$ hartree and $<10^{-5}$ in the maximal wave-function difference. The initial states chosen in calculations are the 6th, 14th, and 15th excited states. Their ionization potentials are $\mathrm{Ip}=1.112$, $0.776,0.633$ hartree, respectively, which were converged in the grid parameters $<10^{-3}$ hartree. The isosurface plots for these orbitals are presented in Fig. 6(d) and clearly show a chiral nature (the lack of any reflection or inversion symmetries). Notably, the main text presents numerical results for this model chiral potential even though its orbitals are not highly chiral (see Fig. 6-the orbitals do not exhibit exact reflection or inversion symmetries but are not far from it). In a realistic molecular system with valance orbitals that are localized on the chiral center, the signal is higher. This prediction is supported by numerical calculations from different initial orbitals that show that orbitals with larger chirality lead to larger disparity, as well as in comparison to the case of $\mathrm{CBrClFH}$, which is much more chiral. This example highlights the importance of the chirality of the initial state and not just that of the chiral molecular potential.

\section{DFT-based model for bromochlorofluoromethane}

The DFT-based model for a chiral molecule was constructed using real-space, grid-based, ground-state DFT calculations performed with the octopus code $[47,50,51]$. Calculations were performed using the Perdew-BurkeErnzerhof (PBE) [52] exchange-correlation functional of the generalized gradient approximation (GGA), which was previously shown useful for describing the optical properties in $\mathrm{CBrClFH}$ [53]. The frozen-core approximation was assumed, and inner-core orbitals were treated using norm-conserving pseudopotentials [54], where a total of 26 valence electrons were used in the neutral molecule. Specifically, the $[\mathrm{Ar}] 3 d^{10},[\mathrm{Ne}],[\mathrm{He}]$, and $[\mathrm{He}]$ shells of $\mathrm{Br}, \mathrm{Cl}, \mathrm{F}$, and $\mathrm{C}$ atoms were described with pseudopotentials, respectively. The Kohn-Sham (KS) equations were solved to self-consistency with a tolerance of less than $<10^{-9}$ hartree, and the grid spacing was converged to $\Delta x=0.4$ bohr, such that the total energy per electron was converged to less than $<10^{-4}$ hartree, and the KS highest occupied molecular orbital (HOMO) eigenvalue was converged to less than $<10^{-4}$ hartree.

First, the molecular structure of $\mathrm{CBrClFH}$ was relaxed to less than $<10^{-4}$ hartree/bohr in forces within unpolarized DFT calculations (i.e., neglecting the spin d.o.f.). Second, in this relaxed geometry, spin-polarized DFT calculations were performed on the singly ionized system (the molecular ion). From these calculations, the $\mathrm{KS}$ potential was extracted, which includes contributions from both the external molecular potential and mean-field electronelectron effects. This effective potential has the correct Coulomb tail to describe the single HOMO electron and is completely free of self-interaction for the HOMO orbital. Third, this effective KS potential was diagonalized similarly to the chiral model potential in A. 1, and the TDSE for the HOMO electron was solved as specified in Appendix A. 3. The second enantiomer was found by reflecting the potential and HOMO orbital along the $y z$ plane. Figure 7 describes the effective KS potential and HOMO.
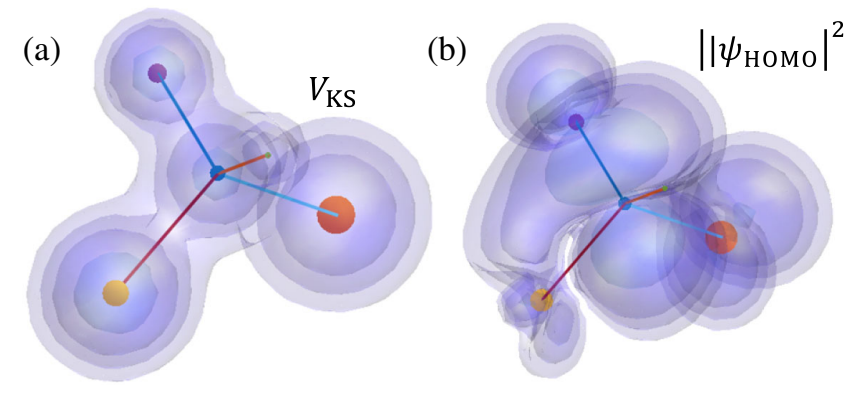

FIG. 7. (a) Effective $\mathrm{KS}$ potential for the molecular $\mathrm{CBrClFH}$ ion. (b) The HOMO orbital for the effective KS potential used in calculations. Blue, purple, yellow, red, and green spheres stand for $\mathrm{C}, \mathrm{F}, \mathrm{Cl}, \mathrm{Br}$, and $\mathrm{H}$ atoms, respectively. Isosurfaces for the potential are $s=0.05,0.1,0.25,\left.0.75|\psi|\right|_{\max } ^{2}$. 


\section{TDSE-Numerical details}

The TDSE defined by the Hamiltonian in Eq. (1) in the main text was solved numerically on a $3 \mathrm{D}$ real-space $\mathrm{L}^{3}$ grid for $L=100$ bohr for the model potential and $L=$ 120 bohr for the DFT-based model. The grid spacing was chosen to be equidistant with $\Delta x=\Delta y=\Delta z=0.2604$, and 0.4 bohr for the model potential and DFT-based model, respectively. The numerical integration was performed using the third-order split step method [55,56], with a time step of $\Delta t=0.02$ a.u. if $\lambda=900 \mathrm{~nm}, 1500 \mathrm{~nm}, 3300 \mathrm{~nm}$, and $\Delta t=0.015$ a.u. if $\lambda=700 \mathrm{~nm}$. An imaginary absorbing potential was used to prevent reflection from the boundaries of the form

$$
V_{a b}(\vec{r})=-i \eta\left(|\vec{r}|-r_{0}\right)^{\gamma} \Theta\left(|\vec{r}|-r_{0}\right),
$$

where for the model potential, $\eta=1.5 \times 10^{-3}, r_{0}=36 \mathrm{bohr}$, and $\gamma=4.3$ for $\psi_{15}$ and $\eta=10^{-3}, r_{0}=33 \mathrm{bohr}$, and $\gamma=4$ for $\psi_{6}$, and for the DFT-based model, $\eta=10^{-3}$, $r_{0}=39 \mathrm{bohr}$, and $\gamma=3.2$. The induced polarization was calculated according to Eq. (2) in the main text on a twice-reduced time grid (i.e., every other time step), and the dipole acceleration was calculated directly using a fifthorder finite difference approximation for the second-order time derivative:

$$
\vec{a}(t)=\frac{d^{2}}{d t^{2}} \vec{P}_{\text {tot }}(t) .
$$

\section{Orientation averaging-Numerical details}

Orientation averaging for the models described in Appendixes A. 1 and A. 2 was achieved by repeatedly solving the TDSE for varying beam propagation orientations and realigning the calculated induced polarization along the correct axis with rotation matrices. This procedure was used for 24 major alignments along the three Cartesian axes, which were prechosen such that, for each orientation $\Omega$ in the ensemble, the inverted and reflected orientations $\Omega^{*}$ also exist, which was verified against a 48-orientation ensemble that upheld similar constraints.

Two independent variables are required in order to define the orientation of a rigid body in 3D. We used a normalized vector $\vec{v}$, which defines the $z$ axis in the frame of the propagating beams in terms of the Cartesian coordinates $x^{\prime}$, $y^{\prime}, z^{\prime}$ in the molecular frame of reference [which corresponds to the potential form in Eq. (A1), or the effective KS potential for the DFT-based model], accompanied by an angle $0<\theta \leq 2 \pi$ that defines the relative rotation about the $\vec{v}$ axis (such that $\theta=0^{0}$ corresponds to the $x$ axis in the beam's frame of reference). The orientations are given by all permutations of the angles $\theta=0^{\circ}, 90^{\circ}, 180^{\circ}, 270^{\circ}$, and $\vec{v}^{\prime}= \pm \hat{x}^{\prime}, \pm \hat{y}^{\prime}, \pm \hat{z}^{\prime}$, which altogether span 24 rigid-body orientations, and the 48-orientation ensemble against which convergence was attained was additionally spanned by the vectors $\vec{v}^{\prime}=\left( \pm \hat{x}^{\prime} \pm \hat{y}^{\prime} \pm \hat{z}^{\prime}\right)$.
Convergence is tested with respect to the amount of orientations and is converged up to $3 \%$ in chiral signal strength [i.e., the chiral signal per harmonic changes, on average, by $3 \%$ between the 24 - and 48 -orientation ensembles; see Fig. 8(a)] and up to $15 \%$ in the ratio of the chiral-tonearby-achiral harmonic emission, i.e., the relative yields of $2 n / 2 n+1$, even/odd, harmonic orders for integer $n$ [see Fig. 8(b)]. This relatively rapid convergence can be explained as follows:

(1) The grid is specifically chosen to hasten the convergence of the DS selection rule-the mechanism relies on interference between the inverted molecular orientations, so a clever grid choice reduces the amount of orientations required for convergence.

(2) The chiral signal is based on intense electric-dipole transitions. This case is very different from standard magnetic effects that lead to small signals, where mild changes to the harmonic yield can drastically change the signal strength. Consider the following examplea reduction in the intensity of a harmonic by $10 \%$ (i.e., due to some unaccounted for orientations) only changes the chiral/achiral signal from $96.3 \%$ to $95.5 \%$. By contrast, in a magnetic-dipole-based small signal (standard cHHG), a 5\% chiral/achiral signal will change to $0 \%$ (no chiral signal) if the intensity is changed by the same mere $10 \%$ because the achiral signal is much larger than the chiral one. This result means that such techniques require very dense angular grids to reach convergence compared to our scheme.

(3) The pump field spans all 3D coordinate space; thus, even a single orientation excites all three spatial axes in the molecular reference frame [see Fig. 8(c) for illustration of the 48-orientaion ensemble pumpfield Lissajou curves], meaning less orientations are required to probe the full molecular response.

\section{Selection rules derivation for the DSs in schemes (A)-(C)}

For self-completeness, we derive the selection rules for the three DSs used in the main text, following the derivation in Ref. [35]. First, we expand the nonlinear polarization $\vec{P}_{\text {tot }}(t)$ from Eq. (3) in the main text to a Fourier series:

$$
\vec{P}_{\text {tot }}(t)=\sum_{n} \vec{F}_{n} \exp (i n \omega t),
$$

where the coefficients $\vec{F}_{n}$ are complex numbers, $n$ is any integer that represents the $n$th harmonic, and $\omega$ is the optical frequency. This expansion is justified in the Floquet limit, where the HHG process is time periodic and only discrete harmonics are emitted. Following the proofs in Ref. [35], a DS of the full Hamiltonian is also necessarily a DS of the induced polarization in Eq. (A4). Using this rule, one can derive the constraints on the emitted frequency components $\vec{F}_{n}$ by enforcing the invariance of $\vec{P}_{\text {tot }}(t)$ under the DSs, 
(c) Lissajou electric field in scheme (C) in molecular

(a) Chiral/achiral signal per even harmonic

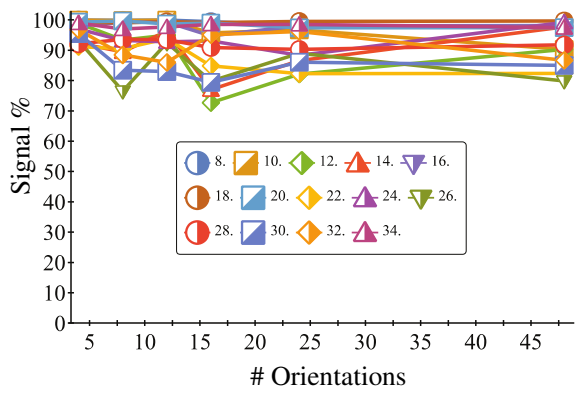

(b) Ratio of chiral to achiral harmonic yield

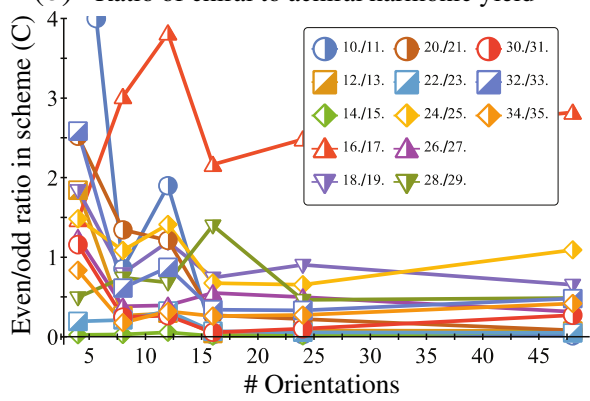

reference frame for the 48-orientation ensemble

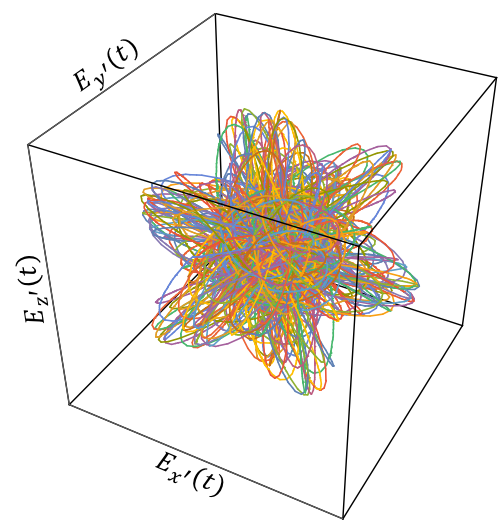

FIG. 8. Convergence with respect to angular grid density in scheme (C) for the DFT-based model, with the same parameters as Fig. 5. (a) Chiral/achiral signal per harmonic order vs orientations. (b) Chiral even harmonic emission ratio to nearby odd achiral harmonic emission. The average ratio is 0.5 for this particular parameter choice. (c) Exemplary representation of the 48-orientation ensemble - the Lissajou curves of the 48 different orientations of pump fields used in the molecular reference frame. As seen, the electric field drives all three spatial axes, even from a single orientation.

which leads to eigenvalue problems in the frequency domain. For $\hat{\sigma}_{x z}$ presented in scheme (A), this means

$$
\sum_{n} \vec{F}_{n} \exp (i n \omega t)=\sum_{n} \hat{\sigma}_{x z} \cdot \vec{F}_{n} \exp (i n \omega t)
$$

Equation (A5) can be simplified to the following constraint for all harmonic orders:

$$
\hat{\sigma}_{x z} \cdot \vec{F}_{n}=\vec{F}_{n} .
$$

Assuming a general form for $\vec{F}_{n}$,

$$
\vec{F}_{n}=\left(F_{n, x}, F_{n, y}, F_{n, z}\right),
$$

Eq. (A6) leads to the constraint $F_{n, y}=0$. This result means that if $\hat{\sigma}_{x z}$ is upheld in the Hamiltonian, no microscopic $y$-polarized harmonics are emitted.
Similarly, we derive the constraints for $\hat{Z}=\hat{\sigma}_{x z} \cdot \hat{\tau}_{2}$ DS used in scheme (B):

$$
\begin{aligned}
\sum_{n} \vec{F}_{n} \exp (\text { in } \omega t) & =\sum_{n} \hat{\sigma}_{x z} \cdot \hat{\tau}_{2} \cdot \vec{F}_{n} \exp (i n \omega t) \\
& =\sum_{n} \hat{\sigma}_{x z} \cdot \vec{F}_{n} \exp (i n \omega(t-T / 2)),
\end{aligned}
$$

leading to the selection rules where $F_{y}$ emission is only allowed for odd harmonics, and $F_{x, z}$ emission is only allowed for even harmonics.

Lastly, the constraints for $\hat{F}=\hat{\imath} \cdot \hat{\tau}_{2}$ DS used in scheme (C) are

$$
\begin{aligned}
\sum_{n} \vec{F}_{n} \exp (\text { in } \omega t) & =\sum_{n} \hat{\imath} \cdot \hat{\tau}_{2} \cdot \vec{F}_{n} \exp (\text { in } \omega t) \\
& =\sum_{n}-\vec{F}_{n} \exp (\text { in } \omega(t-T / 2))
\end{aligned}
$$
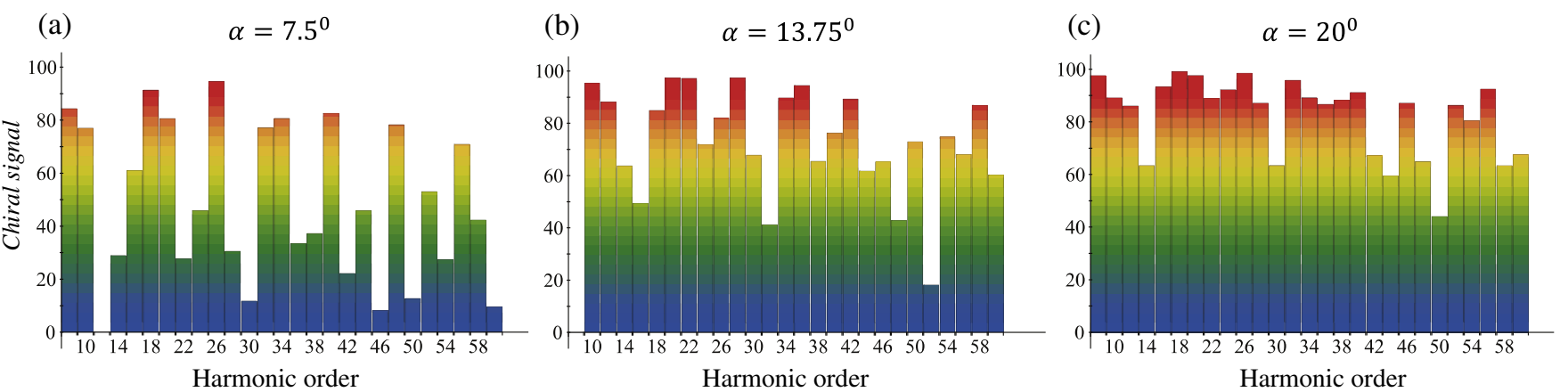

FIG. 9. Dynamical inversion symmetry-breaking-based chiral selectivity in HHG from DFT-based model calculations for CBrClFH. (a)-(c) Chiral signal per harmonic order defined through the harmonic integrated $y$-polarized yield for fundamental wavelength $\lambda=3300 \mathrm{~nm}$, frequency ratios $3 \omega-5 \omega$ in the pump field, $I_{\max }=1.2 \times 10^{13} \mathrm{~W} / \mathrm{cm}^{2}, \phi=\pi / 2, \Delta=1, \varepsilon_{1}=1, \varepsilon_{3}=1$, for $\alpha=7.5^{0}, 13.75^{\circ}, 20^{0}$, and a trapezoidal envelope with four-cycle turn-on/off and three-cycle flattop. 
leading to selection rules where no even harmonic emission is allowed. Because these DSs involve a reflection operation (inversion is a product of reflection and a rotation), they are upheld in achiral media and break in chiral media, emitting a nearly background-free chiral signal.

Notably, one may also consider time-reversal DSs for chiral spectroscopy [35]. However, these DSs lead to linear polarization restrictions that do not allow background-free detection when broken (because the selection rules do not lead to forbidden emission). Furthermore, time reversal is also slightly broken due to strong-field ionization, which reduces the signal-to-noise ratio. Hence, we do not consider these DSs for chiral spectroscopy.

\section{Numerical calculations for different $\alpha$ values and bicircular beams}

We present here calculations for scheme (C) for the DFTbased model of $\mathrm{CBrClFH}$ under several parameter settings that are different than those presented in Figs. 4 and 5 in the main text. Figure 9 presents the chiral signal per harmonic order for several opening angles $\alpha$ but for $\varepsilon_{1}=\varepsilon_{3}=1$, i.e., a bicircular beam (whereas in Figs. 4 and 5 in the main text, results are presented for a bi-elliptical beam). The same qualitative scaling of the chiral signal is observed for $\alpha \rightarrow 0$.

\section{Numerical model for strong-field HHG calculations in propylene oxide}

The high-order harmonic response of a gas-phase ensemble of randomly oriented propylene oxide molecules was evaluated using the method described in Ref. [30], for a single ionization-recombination channel. The harmonic intensity is given by [34]

$$
I_{n}=(n \omega)^{4}|\vec{D}(n \omega)|^{2},
$$

where $\omega$ is the fundamental frequency, $n$ is the harmonic number, and $\vec{D}$ is the frequency-domain dipole induced by the strong field, which results from coherently adding the contributions from all possible molecular orientations:

$$
\vec{D}(n \omega)=\int d \Omega \int d \beta \vec{D}_{\Omega \beta}(n \omega),
$$

where $\vec{D}_{\Omega \beta}$ is the harmonic dipole associated with a given molecular orientation (denoted by two parameters, $\Omega$ and $\beta$ ). The integration over the solid angle $\Omega$ was calculated using the Lebedev quadrature [57] of order 17, i.e., employing 110 Lebedev points. For each Lebedev point, the integration over $\beta$ was performed within the trapezoid method, using 40 different geometries. The single-molecule dipole response $\vec{D}_{\Omega \beta}$ associated with a given orientation was evaluated using the saddle-point method for HHG [34]. Within this method, $\vec{D}_{\Omega \beta}$ is factorized into three terms:

$$
\vec{D}_{\Omega \beta}(n \omega)=a_{\Omega \beta}^{\mathrm{ion}}(n \omega) \cdot a_{\Omega \beta}^{\mathrm{prop}}(n \omega) \cdot \vec{a}_{\Omega \beta}^{\mathrm{rec}}(n \omega),
$$

which are associated with strong-field ionization, propagation, and radiative recombination, respectively. Strong-field ionization amplitudes were evaluated using the following expression:

$$
\begin{aligned}
a_{\Omega \beta}^{\text {ion }}= & \left(\frac{2 \pi}{i \partial^{2} S\left(t_{r}, t_{i}, \vec{p}\right) / \partial t_{i}^{2}}\right)^{1 / 2} \exp \left(-i S\left(t_{i}^{\prime}, t_{i}, \vec{p}\right)\right) \\
& \vec{E}\left(t_{i}^{\prime}\right) \cdot \vec{d}_{\Omega \beta}^{\text {ion }}\left(t_{i}^{\prime}\right),
\end{aligned}
$$

where $t_{i}=t_{i}^{\prime}+i t_{i}^{\prime \prime}$ is the complex ionization time, $\vec{p}$ is the canonical momentum [related to the kinetic momentum, $\vec{k}(t)=\vec{p}+\vec{A}(t)$ ], $\vec{A}(t)$ is the vector potential [related to the time-dependent electric field $\left.\vec{E}(t)=-\partial_{t} \vec{A}(t)\right], \vec{d}_{\Omega \beta}^{\text {ion }}\left(t_{i}^{\prime}\right)$ is the dipole-transition amplitude from the initial state to the Volkov state with kinetic momentum $\operatorname{Re}\left\{\vec{k}\left(t_{i}^{\prime}\right)\right\}$, and

$$
S\left(t, t^{\prime}, \vec{p}\right)=\frac{1}{2} \int_{t^{\prime}}^{t}(\vec{p}+\vec{A}(\tau))^{2} d \tau+I_{p}\left(t-t^{\prime}\right),
$$

where $I_{p}$ is the ionization potential. For the single-channel response considered here, propagation amplitudes can be written as

$$
a_{\Omega \beta}^{\mathrm{prop}}=\left(\frac{2 \pi}{i\left(t_{r}-t_{i}\right)}\right)^{3 / 2} \exp \left(-i S\left(t_{r}^{\prime}, t_{i}^{\prime}, \vec{p}\right)\right)
$$

where $t_{r}=t_{r}^{\prime}+i t_{r}^{\prime \prime}$ is the complex recombination time. Recombination amplitudes are given by

$$
\begin{aligned}
\vec{a}_{\Omega \beta}^{\mathrm{rec}}= & \left(\frac{2 \pi}{i \partial^{2} S\left(t_{r}, t_{i}, \vec{p}\right) / \partial t_{r}^{2}}\right)^{1 / 2} \exp \left(-i S\left(t_{r}, t_{r}^{\prime}, \vec{p}\right)+i n \omega t_{r}\right) \\
& \vec{d}_{\Omega \beta}^{\mathrm{rec}}\left(\vec{k}\left(t_{r}^{\prime}\right)\right),
\end{aligned}
$$

where $\vec{d}_{\Omega \beta}^{\text {rec }}$ is the corresponding photorecombination matrix element. These matrix elements were computed within the static-exchange DFT method [58-61], as in Ref. [30].

[1] M. Kenji, Bioactive Natural Products and Chirality, Chirality $\mathbf{2 3}, 449$ (2011).

[2] U. J. Meierhenrich, Amino Acids and the Asymmetry of Life, Eur. Rev. 21, 190 (2013).

[3] W. H. Brooks, W. C. Guida, and K. G. Daniel, The Significance of Chirality in Drug Design, and Development, Curr. Top. Med. Chem. 11, 760 (2011).

[4] P. Y. Bruice, Organic Chemistry, 5th ed. (Prentice-Hall, Englewood Cliffs, NJ, 2006).

[5] N. Berova, P. L. Polavarapu, K. Nakanishi, and R. W. Woody, Comprehensive Chiroptical Spectroscopy: Applications in Stereochemical Analysis of Synthetic Compounds, Natural 
Products, and Biomolecules (John Wiley \& Sons, New York, 2012).

[6] E. Castiglioni, S. Abbate, F. Lebon, and G. Longhi, Chiroptical Spectroscopic Techniques Based on Fluorescence, Methods Appl. Fluoresc. 2, 024006 (2014).

[7] G. J. Simpson, Molecular Origins of the Remarkable Chiral Sensitivity of Second-Order Nonlinear Optics, Chem. Phys. Chem. 5, 1301 (2004).

[8] M. A. Belkin and Y. R. Shen, Non-Linear Optical Spectroscopy as a Novel Probe for Molecular Chirality, Int. Rev. Phys. Chem. 24, 257 (2005).

[9] P. Fischer, D. S. Wiersma, R. Righini, B. Champagne, and A. D. Buckingham, Three-Wave Mixing in Chiral Liquids, Phys. Rev. Lett. 85, 4253 (2000).

[10] A. F. Ordonez and O. Smirnova, Generalized Perspective on Chiral Measurements without Magnetic Interactions, Phys. Rev. A 98, 063428 (2018).

[11] N. Böwering, T. Lischke, B. Schmidtke, N. Müller, T. Khalil, and U. Heinzmann, Asymmetry in Photoelectron Emission from Chiral Molecules Induced by Circularly Polarized Light, Phys. Rev. Lett. 86, 1187 (2001).

[12] A. Comby, S. Beaulieu, M. Boggio-Pasqua, D. Descamps, F. Légaré, L. Nahon, S. Petit, B. Pons, B. Fabre, Y. Mairesse, and V. Blanchet, Relaxation Dynamics in Photoexcited Chiral Molecules Studied by Time-Resolved Photoelectron Circular Dichroism: Toward Chiral Femtochemistry, J. Phys. Chem. Lett. 7, 4514 (2016).

[13] B. Ritchie, Theory of the Angular Distribution of Photoelectrons Ejected from Optically Active Molecules and Molecular Negative Ions, Phys. Rev. A 13, 1411 (1976).

[14] I. Powis, Photoelectron Circular Dichroism of the Randomly Oriented Chiral Molecules Glyceraldehyde and Lactic Acid, J. Chem. Phys. 112, 301 (2000).

[15] C. S. Lehmann, N. B. Ram, I. Powis, and M. H. M. Janssen, Imaging Photoelectron Circular Dichroism of Chiral Molecules by Femtosecond Multiphoton Coincidence Detection, J. Chem. Phys. 139, 234307 (2013).

[16] C. Lux, M. Wollenhaupt, T. Bolze, Q. Liang, J. Köhler, C. Sarpe, and T. Baumert, Circular Dichroism in the Photoelectron Angular Distributions of Camphor and Fenchone from Multiphoton Ionization with Femtosecond Laser Pulses, Angew. Chem., Int. Ed. Engl. 51, 5001 (2012).

[17] S. Beaulieu, A. Ferré, R Géneaux, R. Canonge, D. Descamps, B. Fabre, N. Fedorov, F. Légaré, S. Petit, T. Ruchon, V. Blanchet, Y. Mairesse, and B. Pons, Universality of Photoelectron Circular Dichroism in the Photoionization of Chiral Molecules, New J. Phys. 18, 102002 (2016).

[18] M. Pitzer, M. Kunitski, A. S. Johnson, T. Jahnke, H. Sann, F. Sturm, L. P. H. Schmidt, H. Schmidt-Böcking, R. Dörner, J. Stohner, J. Kiedrowski, M. Reggelin, S. Marquardt, A. Schießer, R. Berger, and M. S. Schöffler, Direct Determination of Absolute Molecular Stereochemistry in Gas Phase by Coulomb Explosion Imaging, Science 341, 1096 (2013).

[19] P. Herwig, K. Zawatzky, M. Grieser, O. Heber, B. JordonThaden, C. Krantz, O. Novotný, R. Repnow, V. Schurig, D. Schwalm, Z. Vager, A. Wolf, O. Trapp, and H. Kreckel, Imaging the Absolute Configuration of a Chiral Epoxide in the Gas Phase, Science. 342, 1084 (2013).
[20] D. Patterson, M. Schnell, and J. M. Doyle, EnantiomerSpecific Detection of Chiral Molecules via Microwave Spectroscopy, Nature (London) 497, 475 (2013).

[21] S. Eibenberger, J. Doyle, and D. Patterson, EnantiomerSpecific State Transfer of Chiral Molecules, Phys. Rev. Lett. 118, 123002 (2017).

[22] H. Rhee, Y.-G. June, J.-S. Lee, K.-K. Lee, J.-H. Ha, Z. H. Kim, S.-J. Jeon, and M. Cho, Femtosecond Characterization of Vibrational Optical Activity of Chiral Molecules, Nature (London) 458, 310 (2009).

[23] S. Beaulieu, A. Comby, B. Fabre, D. Descamps, A. Ferre, G. Garcia, R. Geneaux, F. Legare, L. Nahon, S. Petit, T. Ruchon, B. Pons, V. Blanchet, and Y. Mairesse, Probing Ultrafast Dynamics of Chiral Molecules Using TimeResolved Photoelectron Circular Dichroism, Faraday Discuss. 194, 325 (2016).

[24] R. E. Goetz, T. A. Isaev, B. Nikoobakht, R. Berger, and C. P. Koch, Theoretical Description of Circular Dichroism in Photoelectron Angular Distributions of Randomly Oriented Chiral Molecules after Multi-Photon Photoionization, J. Chem. Phys. 146, 024306 (2017).

[25] S. Beaulieu, A. Comby, A. Clergerie, J. Caillat, D. Descamps, N. Dudovich, B. Fabre, R. Géneaux, F. Légaré, S. Petit, B. Pons, G. Porat, T. Ruchon, R. Taïeb, V. Blanchet, and Y. Mairesse, Attosecond-Resolved Photoionization of Chiral Molecules, Science 358, 1288 (2017).

[26] S. Beaulieu, A. Comby, D. Descamps, B. Fabre, G. A. Garcia, R. Géneaux, A. G. Harvey, F. Légaré, Z. Mašín, and L. Nahon, Photoexcitation Circular Dichroism in Chiral Molecules, Nat. Phys. 14, 484 (2018).

[27] A. G. Harvey, Z. Mašín, and O. Smirnova, General Theory of Photoexcitation Induced Photoelectron Circular Dichroism, J. Chem. Phys. 149, 064104 (2018).

[28] R. Cireasa, A. E. Boguslavskiy, B. Pons, M. C. H. Wong, D. Descamps, S. Petit, H. Ruf, N. Thiré, A. Ferré, J. Suarez, J. Higuet, B. E. Schmidt, A. F. Alharbi, F. Légaré, V. Blanchet, B. Fabre, S. Patchkovskii, O. Smirnova, Y. Mairesse, and V. R. Bhardwaj, Probing Molecular Chirality on a SubFemtosecond Timescale, Nat. Phys. 11, 654 (2015).

[29] O. Smirnova, Y. Mairesse, and S. Patchkovskii, Opportunities for Chiral Discrimination Using High Harmonic Generation in Tailored Laser Fields, J. Phys. B At. Mol. Opt. Phys. 48, 234005 (2015).

[30] D. Ayuso, P. Decleva, S. Patchkovskii, and O. Smirnova, Chiral Dichroism in Bi-Elliptical High-Order Harmonic Generation, J. Phys. B At. Mol. Opt. Phys. 51, 06LT01 (2018).

[31] D. Ayuso, P. Decleva, S. Patchkovskii, and O. Smirnova, Strong-Field Control and Enhancement of Chiral Response in Bi-Elliptical High-Order Harmonic Generation: An Analytical Model, J. Phys. B At. Mol. Opt. Phys. 51, 124002 (2018).

[32] Y. Harada, E. Haraguchi, K. Kaneshima, and T. Sekikawa, Circular Dichroism in High-Order Harmonic Generation from Chiral Molecules, Phys. Rev. A 98, 021401(R) (2018).

[33] D. Baykusheva and H. J. Wörner, Chiral Discrimination through Bielliptical High-Harmonic Spectroscopy, Phys. Rev. X 8, 031060 (2018).

[34] T. Schultz and M. Vrakking, Attosecond and XUV Physics: Ultrafast Dynamics and Spectroscopy (Wiley, New York, 2014). 
[35] O. Neufeld, D. Podolsky, and O. Cohen, Floquet Group Theory and Its Application to Selection Rules in Harmonic Generation, Nat. Commun. 10, 405 (2019).

[36] N. Ben-Tal, N. Moiseyev, and A. Beswick, The Effect of Hamiltonian Symmetry on Generation of Odd and Even Harmonics, J. Phys. B 26, 3017 (1993).

[37] O. E. Alon, V. Averbukh, and N. Moiseyev, Selection Rules for the High Harmonic Generation Spectra, Phys. Rev. Lett. 80, 3743 (1998).

[38] A. Fleischer, O. Kfir, T. Diskin, P. Sidorenko, and O. Cohen, Spin Angular Momentum and Tunable Polarization in High-Harmonic Generation, Nat. Photonics 8, 543 (2014).

[39] O. Kfir, P. Grychtol, E. Turgut, R. Knut, D. Zusin, D. Popmintchev, T. Popmintchev, H. Nembach, J. M. Shaw, A. Fleischer, H. Kapteyn, M. Murnane, and O. Cohen, Generation of Bright Phase-Matched Circularly-Polarized Extreme Ultraviolet High Harmonics, Nat. Photonics 9, 99 (2015).

[40] D. Baykusheva, M. S. Ahsan, N. Lin, and H. J. Wörner, Bicircular High-Harmonic Spectroscopy Reveals Dynamical Symmetries of Atoms and Molecules, Phys. Rev. Lett. 116, 123001 (2016).

[41] J. B. Bertrand, H. J. Wörner, H.-C. Bandulet, É. Bisson, M. Spanner, J.-C. Kieffer, D. M. Villeneuve, and P. B. Corkum, Ultrahigh-Order Wave Mixing in Noncollinear High Harmonic Generation, Phys. Rev. Lett. 106, 023001 (2011).

[42] C. M. Heyl, P. Rudawski, F. Brizuela, S. N. Bengtsson, J. Mauritsson, and A. L'Huillier, Macroscopic Effects in Noncollinear High-Order Harmonic Generation, Phys. Rev. Lett. 112, 143902 (2014).

[43] D. D. Hickstein, F. J. Dollar, P. Grychtol, J. L. Ellis, R. Knut, C. Hernández-García, D. Zusin, C. Gentry, J. M. Shaw, T. Fan, K. M. Dorney, A. Becker, A. Jaroń-Becker, H. C. Kapteyn, M. M. Murnane, and C. G. Durfee, Non-Collinear Generation of Angularly Isolated Circularly Polarized High Harmonics, Nat. Photonics 9, 743 (2015).

[44] Y. Tang and A.E. Cohen, Optical Chirality and Its Interaction with Matter, Phys. Rev. Lett. 104, 163901 (2010).

[45] Y. Tang and A. E. Cohen, Enhanced Enantioselectivity in Excitation of Chiral Molecules by Superchiral Light, Science 332, 333 (2011).

[46] O. Neufeld and O. Cohen, Optical Chirality in Nonlinear Optics: Application to High Harmonic Generation, Phys. Rev. Lett. 120, 133206 (2018).

[47] A. Castro, H. Appel, M. Oliveira, C. A. Rozzi, X. Andrade, F. Lorenzen, M. A. L. Marques, E. K. U. Gross, and A. Rubio, Octopus: A Tool for the Application of TimeDependent Density Functional Theory, Phys. Status Solidi 243, 2465 (2006).

[48] X. Andrade, D. Strubbe, U. De Giovannini, H. Larsen, M. J. T. Oliveira, J. Alberdi-rodriguez, A. Varas, I. Theophilou, N. Helbig, M. J. Verstraete, L. Stella, F. Nogueira, A. Castro, M. A. L. Marques, and A. Rubio, Real-Space Grids and the Octopus Code as Tools for the Development of New Simu- lation Approaches for Electronic Systems, Phys. Chem. Chem. Phys. 17, 31371 (2015).

[49] D. Ayuso, O. Neufeld, A. F. Ordonez, P. Decleva, G. Lerner, O. Cohen, M. Ivanov, and O. Smirnova, Locally and Globally Chiral Fields for Ultimate Control of Chiral Light Matter Interaction, arXiv:1809.01632v1; arXiv:1809.01632.

[50] M. A. L. Marques, A. Castro, G. F. Bertsch, and A. Rubio, Octopus: A First-Principles Tool for Excited Electron-Ion Dynamics, Comput. Phys. Commun. 151, 60 (2003).

[51] X. Andrade, D. Strubbe, U. De Giovannini, A. H. Larsen, M. J. T. Oliveira, J. Alberdi-Rodriguez, A. Varas, I. Theophilou, N. Helbig, M. J. Verstraete, L. Stella, F. Nogueira, A. Aspuru-Guzik, A. Castro, M. A. L. Marques, and A. Rubio, Real-Space Grids and the Octopus Code as Tools for the Development of New Simulation Approaches for Electronic Systems, Phys. Chem. Chem. Phys. 17, 31371 (2015).

[52] J. P. Perdew, K. Burke, and M. Ernzerhof, Generalized Gradient Approximation Made Simple, Phys. Rev. Lett. 77, 3865 (1996).

[53] J. Autschbach, S. Patchkovskii, T. Ziegler, S. J. A. van Gisbergen, and E. Jan Baerends, Chiroptical Properties from Time-Dependent Density Functional Theory. II. Optical Rotations of Small to Medium Sized Organic Molecules, J. Chem. Phys. 117, 581 (2002).

[54] M. Schlipf and F. Gygi, Optimization Algorithm for the Generation of ONCV Pseudopotentials, Comput. Phys. Commun. 196, 36 (2015).

[55] J. A. Fleck, J. R. Morris, and M. D. Feit, Time-Dependent Propagation of High Energy Laser Beams through the Atmosphere, Appl. Phys. 10, 129 (1976).

[56] M. D. Feit, J. A. Fleck, and A. Steiger, Solution of the Schrödinger Equation by a Spectral Method, J. Comput. Phys. 47, 412 (1982).

[57] V. I. Lebedev and D. N. Laikov, A Quadrature Formula for the Sphere of the 131st Algebraic Order of Accuracy, Dokl. Math. 59, 477 (1999).

[58] D. Toffoli, M. Stener, G. Fronzoni, and P. Decleva, Convergence of the Multicenter B-Spline DFT Approach for the Continuum, Chem. Phys. 276, 25 (2002).

[59] S. Turchini, N. Zema, G. Contini, G. Alberti, M. Alagia, S. Stranges, G. Fronzoni, M. Stener, P. Decleva, and T. Prosperi, Circular Dichroism in Photoelectron Spectroscopy of Free Chiral Molecules: Experiment and Theory on Methyl-Oxirane, Phys. Rev. A 70, 014502 (2004).

[60] M. Stener, G. Fronzoni, D. Di Tommaso, and P. Decleva, Density Functional Study on the Circular Dichroism of Photoelectron Angular Distribution from Chiral Derivatives of Oxirane, J. Chem. Phys. 120, 3284 (2004).

[61] S. Stranges, S. Turchini, M. Alagia, G. Alberti, G. Contini, P. Decleva, G. Fronzoni, M. Stener, N. Zema, and T. Prosperi, Valence Photoionization Dynamics in Circular Dichroism of Chiral Free Molecules: The Methyl-Oxirane, J. Chem. Phys. 122, 244303 (2005). 